\title{
Article \\ A Novel Numerical Approach in Solving Fractional Neutral Pantograph Equations via the ARA Integral Transform
}

\author{
Aliaa Burqan *, Rania Saadeh (D) and Ahmad Qazza (D) \\ Department of Mathematics, Zarqa University, Zarqa 13132, Jordan; rsaadeh@zu.edu.jo (R.S.); \\ aqazza@zu.edu.jo (A.Q.) \\ * Correspondence: aliaaburqan@zu.edu.jo
}

check for updates

Citation: Burqan, A.; Saadeh, R.; Qazza, A. A Novel Numerical Approach in Solving Fractional Neutral Pantograph Equations via the ARA Integral Transform. Symmetry 2022, 14, 50. https:// doi.org/10.3390/sym14010050

Academic Editor: Louis H. Kauffman

Received: 21 November 2021

Accepted: 15 December 2021

Published: 31 December 2021

Publisher's Note: MDPI stays neutral with regard to jurisdictional claims in published maps and institutional affiliations.

Copyright: (C) 2021 by the authors. Licensee MDPI, Basel, Switzerland. This article is an open access article distributed under the terms and conditions of the Creative Commons Attribution (CC BY) license (https:// creativecommons.org/licenses/by/ $4.0 /)$.

\begin{abstract}
In this article, a new, attractive method is used to solve fractional neutral pantograph equations (FNPEs). The proposed method, the ARA-Residual Power Series Method (ARA-RPSM), is a combination of the ARA transform and the residual power series method and is implemented to construct series solutions for dispersive fractional differential equations. The convergence analysis of the new method is proven and shown theoretically. To validate the simplicity and applicability of this method, we introduce some examples. For measuring the accuracy of the method, we make a comparison with other methods, such as the Runge-Kutta, Chebyshev polynomial, and variational iterative methods. Finally, the numerical results are demonstrated graphically.
\end{abstract}

Keywords: Caputo fractional derivative; fractional neutral pantograph equations; power series; the ARA transform

\section{Introduction}

Fractional calculus is an area of applied mathematics that deals with integrals and derivatives of fractional orders. Over the last decades, it has turned out that many phenomena in physics, chemistry, engineering, and other fields of science can be expressed accurately by models using mathematical tools through fractional orders. For example, fractional derivatives have been used to describe chemical processes, mathematical biology, and many other problems in physics and engineering. More specific information about differential integral equations and fractional calculus can be found in [1-12].

Mostly, nonlinear fractional differential equations do not have exact solutions; so many numerical and analytical methods have been introduced and implemented to obtain their approximate solutions. For example, the Adomian decomposition method (ADM) [13] was applied to solve nonlinear fractional diffusion and wave equations [14,15], and the homotopy perturbation method (HPM) was constructed in [16] and was used to study dissipative nonplanar solitons in an electronegative complex plasma [17], etc. [18-23]. Nowadays the residual power series method (RPSM) is used to construct power series solutions of differential equations without linearization, discretization, or perturbation [24-26].

Recently, a new integral transform called the ARA transform was implemented successfully to solve some ordinary and partial differential equations [27]. Moreover, an attractive formula for the ARA transform of the Caputo fractional derivatives was obtained in [28] and used to create series solutions for some families of fractional differential equations.

Conversely, some authors combined two powerful methods to obtain new methods to solve fractional differential equations; Mahmood et al. [29] used the Laplace-Adomian decomposition method (LADM), a combination of the Laplace transform and the ADM; Kumar et al. [30] introduced the homotopy perturbation transform method (HPTM) and combined the Laplace transform with the HPM; Eriqat et al. [31] constructed the Laplaceresidual power series method (LRPSM), combining the Laplace transform with the RPSM, and so on [32-34]. 
Fractional delay differential equations appear in many applications, such as long transmission lines, automatic control, biology, and economy [35]. The fractional pantograph equation (FPE) is one of the fractional delay differential equations that plays a vital role in explaining diverse phenomena [36], so it has attracted the attention of many researchers. Balachandran and Kiruthika [34] studied the existence of the solutions of nonlinear FPEs. Rahimkhani, Ordokhani, and Babolian [37] utilized the generalized fractionalorder Bernoulli wavelet to obtain the numerical solution of FPEs, whereas Eriqat et al. [31] introduced accurate and numerical series solutions to the FNPEs by using the LRPSM. However, only a few articles are dedicated to the approximate solution of FNPEs.

In this paper, we consider the FNPE subject at the initial condition

$$
\begin{gathered}
D^{\alpha} g(t)=a g(\beta t)+b D^{\alpha} g(\gamma t)+c g(\lambda t)+f(t) \\
g(0)=g_{0}
\end{gathered}
$$

where $a, b, c \in \mathbb{R}, 0<\alpha \leq 1,0<\beta, \gamma, \lambda<1, D^{\alpha}$ is the Caputo fractional derivative of order $\alpha$ and $f(t)$ has a fractional power series expansion.

Throughout this work, we present an efficient analytical technique to construct series solutions of fractional differential equations. The new method named the "ARA-Residual Power Series Method" (ARA-RPSM) is based on combining the ARA transform and the RPSM into a new vision. To illustrate the efficiency of this method, it is applied to the FNPEs to construct the so-called ARA-RPS solution.

\section{Definitions and Theorems}

This section consists of two parts. In the first one, we introduce some basic definitions of fractional operators and the ARA transform with some properties that are essential in our work.

In the second part, we introduce some new results related to the ARA transform, and the fractional derivatives are introduced and proven.

\subsection{Preliminaries}

Definition 1. [9] The Riemann-Liouville fractional integral of the function $g(t)$ of order $\alpha>0$ is defined by

$$
I_{t}^{\alpha} g(t)=\frac{1}{\Gamma(\alpha)} \int_{a}^{t}(t-\tau)^{\alpha-1} g(\tau) d \tau
$$

Definition 2. [9] The Caputo fractional derivative of the function $g(t)$ of order $\alpha>0$ is defined by

$$
D^{\alpha} g(t)= \begin{cases}\frac{1}{\Gamma(n-\alpha)} \int_{0}^{t} \frac{g^{(n)}(\tau)}{(t-\tau)^{\alpha+1-n}} d \tau, & n-1<\alpha<n \\ g^{(n)}(t) & \alpha=n\end{cases}
$$

Definition 3. [27] The ARA transform of order $n$ of the continuous function $g(t)$ on the interval $(0, \infty)$ is defined by

$$
\mathcal{G}_{n}[g(t)](s)=s \int_{0}^{\infty} t^{n-1} e^{-s t} g(t) d t, s>0 .
$$

Theorem 1. [27] (The sufficient condition for the existence of the ARA transform). If the function $g(t)$ is piecewise continuous in every finite interval $0 \leq t \leq \beta$ and satisfies $\left|t^{n-1} g(t)\right| \leq K e^{\beta t}$, then the ARA transform exists for all $s>\beta$. 
In the following arguments, we present some properties of the ARA transform $[27,28]$ that are needed in our work.

Let $g(t)$ and $h(t)$ be two continuous functions on the interval $(0, \infty)$ in which the ARA transform exists. Then

1. $\mathcal{G}_{n}[\alpha g(t)+\beta h(t)](s)=\alpha \mathcal{G}_{n}[g(t)](s)+\beta \mathcal{G}_{n}[h(t)](s)$,

2. where $\alpha$ and $\beta$ are nonzero constants.

3. $\mathcal{G}_{1}[g(t)](s)=s G(s)$ where $G(s)$ is the Laplace transform of $g(t)$.

4. $\lim _{s \rightarrow \infty} \mathcal{G}_{1}[g(t)](s)=g(0)$.

5. $\mathcal{G}_{1}\left[g^{\prime}(t)\right](s)=s \mathcal{G}_{1}[g(t)](s)-s g(0)$.

6. $\lim _{s \rightarrow \infty} \frac{d}{d s}\left[\frac{\mathcal{G}_{1}[g(t)](s)}{s}\right]=0$.

7. $\mathcal{G}_{2}[g(t)](s)=-s \frac{d}{d s}\left(\frac{\mathcal{G}_{1}[g(t)](s)}{s}\right)$.

8. $\mathcal{G}_{2}\left[g^{\prime}(t)\right](s)=-s \frac{d}{d s}\left(\mathcal{G}_{1}[g(t)](s)\right)$.

9. $\mathcal{G}_{2}\left[t^{m}\right](s)=\frac{\Gamma(m+2)}{s^{m+1}}, s>0$.

10. $\mathcal{G}_{n}[g(a t)](s)=\frac{1}{a^{n-1}} \mathcal{G}_{n}[g(t)]\left(\frac{s}{a}\right)$.

\subsection{New Results Related to the ARA Transform}

In the following, we establish new results that are needed to construct the ARA-RPS solutions of fractional differential equations.

Theorem 2. Let $g(t)$ be a continuous function on the interval $(0, \infty)$ in which the ARA transform exists. Then

$$
\lim _{s \rightarrow \infty}\left(\mathcal{G}_{1}[g(t)](s)-s \mathcal{G}_{1}^{\prime}[g(t)](s)\right)=g(0) .
$$

Proof of Theorem 2. Property (2) implies

$$
\begin{aligned}
& \lim _{s \rightarrow \infty}\left(\mathcal{G}_{1}[g(t)](s)-s \mathcal{G}_{1}^{\prime}[g(t)](s)\right)=\lim _{s \rightarrow \infty}\left(s G(s)-s(s G(s))^{\prime}\right) \\
& =\lim _{s \rightarrow \infty}\left(s G(s)-s^{2} G^{\prime}(s)-s G(s)\right)=\lim _{s \rightarrow \infty}-s^{2} G^{\prime}(s) .
\end{aligned}
$$

The proof is completed by showing that

$$
\lim _{s \rightarrow \infty}-s^{2} G^{\prime}(s)=g(0) .
$$

Property (3) implies

$$
\lim _{s \rightarrow \infty} s G(s)=\lim _{s \rightarrow \infty} \mathcal{G}_{1}[g(t)](s)=g(0)
$$

So,

$$
\lim _{s \rightarrow \infty} \frac{G(s)}{\frac{1}{s}}=g(0) .
$$

According to L'Hopital's rule, $\lim _{s \rightarrow \infty} \frac{G^{\prime}(s)}{\frac{-1}{s^{2}}}=\lim _{s \rightarrow \infty}\left(-s^{2} G^{\prime}(s)\right)=g(0)$.

Based on Theorem 2 and using Property (6) of the ARA transform, we reach the following result:

Corollary 1. If $g(t)$ is a continuous function on the interval $(0, \infty)$ in which the ARA transform exists, then

$$
\lim _{s \rightarrow \infty} s \mathcal{G}_{2}[g(t)](s)=g(0)
$$


In the following arguments, we present some results about the ARA transform for the fractional derivatives $D^{\alpha}$.

Lemma 1. [28] The ARA transform of the Caputo fractional derivative of order $\alpha, 0<\alpha \leq 1$ of the function $g(t)$ is given by

$$
\mathcal{G}_{2}\left[D^{\alpha} g(t)\right](s)=\frac{1-\alpha}{s^{2-\alpha}} \mathcal{G}_{1}\left[g^{\prime}(t)\right](s)+\frac{1}{s^{1-\alpha}} \mathcal{G}_{2}\left[g^{\prime}(t)\right](s) .
$$

Lemma 2. Suppose that $g(t)$ is a continuous function on the interval $(0, \infty)$ in which the ARA transform exists and let $0<\alpha \leq 1$, then

i. $\quad \mathcal{G}_{1}\left[D^{n \alpha} g(t)\right](s)=s^{n \alpha} \mathcal{G}_{1}[g(t)](s)-\sum_{k=0}^{n-1} s^{(n-k) \alpha} D^{k \alpha} g(0)$, where $D^{n \alpha}=\underbrace{D^{\alpha} D^{\alpha} \cdots D^{\alpha}}_{n \text {-times }}$

ii. $\quad \mathcal{G}_{2}\left[D^{\alpha} g(t)\right](s)=s^{\alpha} \mathcal{G}_{2}[g(t)](s)-\alpha s^{\alpha-1} \mathcal{G}_{1}[g(t)](s)+(\alpha-1) s^{\alpha-1} g(0)$.

iii. $\quad \mathcal{G}_{2}\left[D^{\alpha} g(a t)\right](s)=\frac{s^{\alpha}}{a} \mathcal{G}_{2}[g(t)]\left(\frac{s}{a}\right)-\alpha s^{\alpha-1} \mathcal{G}_{1}[g(t)]\left(\frac{s}{a}\right)+(\alpha-1) s^{\alpha-1} g(0)$.

Proof Lemma 2. The proof of part (i) can be found in [28] based on Property (2). To prove (ii), Lemma 1, Property (4) and Property (7) yield that

$$
\begin{aligned}
& \mathcal{G}_{2}\left[D^{\alpha} g(t)\right](s)=(1-\alpha) s^{\alpha-1} \mathcal{G}_{1}[g(t)](s)-(1-\alpha) s^{\alpha-1} g(0)-s^{\alpha} \mathcal{G}_{1}^{\prime}[g(t)](s) \\
& =s^{\alpha}\left(\frac{\mathcal{G}_{1}[g(t)](s)}{s}-\mathcal{G}_{1}^{\prime}[g(t)](s)\right)-\alpha s^{\alpha-1} \mathcal{G}_{1}[g(t)](s) \\
& -(1-\alpha) s^{\alpha-1} g(0) \\
& =s^{\alpha}\left(-s \frac{d}{d s}\left(\frac{\mathcal{G}_{1}[g(t)](s)}{s}\right)\right)-\alpha s^{\alpha-1} \mathcal{G}_{1}[g(t)](s) \\
& +(\alpha-1) s^{\alpha-1} g(0) .
\end{aligned}
$$

The desired result is obtained by Property (6).

The proof of (iii) comes directly from (ii) and Property (9).

Theorem 3. Let $g(t)$ be a continuous function on the interval $(0, \infty)$ in which the ARA transform exists. Then

$$
\begin{aligned}
& \mathcal{G}_{2}\left[D^{n \alpha} g(t)\right](s)=s^{n \alpha} \mathcal{G}_{2}[g(t)](s)-n \alpha s^{n \alpha-1} \mathcal{G}_{1}[g(t)](s) \\
& +\sum_{k=0}^{n-1}((n-k) \alpha-1) s^{(n-k) \alpha-1} D^{k \alpha} g(0)
\end{aligned}
$$

Proof Theorem 3. We will use the principle of mathematical induction to obtain the result. For $n=1$, the formula

$$
\mathcal{G}_{2}\left[D^{\alpha} g(t)\right](s)=s^{\alpha} \mathcal{G}_{2}[g(t)](s)-\alpha s^{\alpha-1} \mathcal{G}_{1}[g(t)](s)+(\alpha-1) s^{\alpha-1} g(0),
$$

is true based on part (ii) of Lemma 2.

Assume that the following formula is true for $n=m$ :

$$
\begin{aligned}
& \mathcal{G}_{2}\left[D^{m \alpha} g(t)\right](s)=s^{m \alpha} \mathcal{G}_{2}[g(t)](s)-m \alpha s^{m \alpha-1} \mathcal{G}_{1}[g(t)](s) \\
& +\sum_{k=0}^{m-1}((m-k) \alpha-1) s^{(m-k) \alpha-1} D^{k \alpha} g(0) .
\end{aligned}
$$


We need to prove that the formula is true for $n=m+1$, and Equations (4) and (5) and part (i) of Lemma 2 imply that

$$
\begin{aligned}
& \mathcal{G}_{2}\left[D^{(m+1) \alpha} g(t)\right](s)=\mathcal{G}_{2}\left[D^{\alpha}\left(D^{m \alpha} g(t)\right)\right](s) \\
= & s^{\alpha} \mathcal{G}_{2}\left[D^{m \alpha} g(t)\right](s)-\alpha s^{\alpha-1} \mathcal{G}_{1}\left[D^{m \alpha} g(t)\right](s) \\
+ & (\alpha-1) s^{\alpha-1} D^{m \alpha} g(0) \\
& =s^{\alpha}\left(s^{m \alpha} \mathcal{G}_{2}[g(t)](s)-m \alpha s^{m \alpha-1} \mathcal{G}_{1}[g(t)](s)\right. \\
+ & \left.\sum_{k=0}^{m-1}((m-k) \alpha-1) s^{(m-k) \alpha-1} D^{k \alpha} g(0)\right) \\
- & \alpha s^{\alpha-1}\left(s^{m \alpha} \mathcal{G}_{1}[g(t)](s)-\sum_{k=1}^{m-1} s^{(m-k) \alpha} D^{k \alpha} g(0)\right) \\
+ & (\alpha-1) s^{\alpha-1} D^{m \alpha} g(0) .
\end{aligned}
$$

Therefore,

$$
\begin{aligned}
& \mathcal{G}_{2}\left[D^{(m+1) \alpha} g(t)\right](s) \\
& =s^{(m+1) \alpha} \mathcal{G}_{2}[g(t)](s)-(m+1) \alpha s^{(m+1) \alpha-1} \mathcal{G}_{1}[g(t)](s) \\
& +\sum_{k=0}^{m}((m+1-k) \alpha-1) s^{(m+1-k) \alpha-1} D^{k \alpha} g(0) .
\end{aligned}
$$

The proof is completed.

\section{Fractional Power Series}

Suppose that the fractional power series representation of the function $g(t)$ at $t=0$ has the form [9]

$$
g(t)=\sum_{n=0}^{\infty} a_{n} t^{n \alpha}, 0<m-1<\alpha \leq m, 0 \leq t \leq \beta .
$$

If $g(t)$ and $D^{n \alpha} g(t)$ are continuous on $[0, \beta]$ for $n=0,1,2, \ldots$, then the coefficients $a_{n}$ in the equation has the form

$$
a_{n}=\frac{D^{n \alpha} g(0)}{\Gamma(n \alpha+1)} .
$$

In the following theorem, we present a new fractional Taylor's expansion using the ARA transform, which forms a base for constructing the ARA-RPS solutions of the FNPEs.

Theorem 4. Let $g(t)$ be a continuous function on every finite interval $[0, \beta]$ in which the ARA transform exists and has the fractional power series representation

$$
\mathcal{G}_{2}[g(t)](s)=\sum_{n=0}^{\infty} \frac{h_{n}}{s^{n \alpha+1}} ; 0<\alpha \leq 1 \text { and } s>0 .
$$

Then

$$
h_{n}=(n \alpha+1) D^{n \alpha} g(0)
$$

Proof Theorem 4. Assume that the function $\mathcal{G}_{2}[g(t)](s)$ has the fractional power series represented in Equation (6).

Firstly, if we multiply both sides of Equation (6) by $s$ and take the limit as $s \rightarrow \infty$, then

$$
\lim _{s \rightarrow \infty} s \mathcal{G}_{2}[g(t)](s)=h_{0}+\lim _{s \rightarrow \infty} \sum_{n=1}^{\infty} \frac{h_{n}}{s^{n \alpha}} .
$$


By Corollary 1, we obtain

$$
h_{0}=g(0)
$$

So, Equation (6) becomes

$$
\mathcal{G}_{2}[g(t)](s)=\frac{g(0)}{s}+\sum_{n=1}^{\infty} \frac{h_{n}}{s^{n \alpha+1}}
$$

Similarly, we multiply Equation (7) by $s^{\alpha+1}$ and take the limit as $s \rightarrow \infty$ to obtain $h_{1}$ as follows:

$$
\lim _{s \rightarrow \infty} s^{\alpha+1} \mathcal{G}_{2}[g(t)](s)=\lim _{s \rightarrow \infty} s^{\alpha} g(0)+h_{1}+\lim _{s \rightarrow \infty} \sum_{n=2}^{\infty} \frac{h_{n}}{s^{(n-1) \alpha}} .
$$

Thus,

$$
h_{1}=\lim _{s \rightarrow \infty}\left(s^{\alpha+1} \mathcal{G}_{2}[g(t)](s)-s^{\alpha} g(0)\right)=\lim _{s \rightarrow \infty} s\left(s^{\alpha} \mathcal{G}_{2}[g(t)](s)-s^{\alpha-1} g(0)\right)
$$

Putting $n=1$ in Theorem 3 , we have

$$
\begin{aligned}
& h_{1}=\lim _{s \rightarrow \infty} s\left(\mathcal{G}_{2}\left[s D^{\alpha} g(t)\right](s)+\alpha s^{\alpha-1} \mathcal{G}_{1}[g(t)](s)-\alpha s^{\alpha-1} g(0)\right) \\
& =\lim _{s \rightarrow \infty} s \mathcal{G}_{2}\left[D^{\alpha} g(t)\right](s)+\lim _{s \rightarrow \infty} \alpha\left(s^{\alpha} \mathcal{G}_{1}[g(t)](s)-s^{\alpha} g(0)\right)
\end{aligned}
$$

Lemma 2, part (i) with $n=1$ implies that

$$
h_{1}=\lim _{s \rightarrow \infty} s \mathcal{G}_{2}\left[D^{\alpha} g(t)\right](s)+\alpha \lim _{s \rightarrow \infty} \mathcal{G}_{1}\left[D^{\alpha} g(t)\right](s) .
$$

According to Corollary 1 and Property (3), we obtain

$$
h_{1}=(\alpha+1) D^{\alpha} g(0) \text {. }
$$

Hence, $\mathcal{G}_{2}[g(t)](s)$ can be written as

$$
\mathcal{G}_{2}[g(t)](s)=\frac{g(0)}{s}+\frac{(\alpha+1) D^{\alpha} g(0)}{s^{\alpha+1}}+\frac{h_{2}}{s^{2 \alpha+1}}+\sum_{n=3}^{\infty} \frac{h_{n}}{s^{n \alpha+1}} .
$$

To find $h_{2}$, multiply both sides of Equation (8) by $s^{2 \alpha+1}$ and take the limit as $s \rightarrow \infty$

$$
\lim _{s \rightarrow \infty} s^{2 \alpha+1} \mathcal{G}_{2}[g(t)](s)=\lim _{s \rightarrow \infty} s^{2 \alpha} g(0)+\lim _{s \rightarrow \infty}(\alpha+1) s^{\alpha} D^{\alpha} g(0)+h_{2} .
$$

Thus,

$$
h_{2}=\lim _{s \rightarrow \infty} s\left(s^{2 \alpha} \mathcal{G}_{2}[g(t)](s)-\alpha s^{\alpha-1} D^{\alpha} g(0)-s^{\alpha-1} D^{\alpha} g(0)-s^{2 \alpha-1} g(0)\right)
$$

Again, Theorem 3 with $n=2$ will lead to

$$
\begin{aligned}
& h_{2}=\lim _{s \rightarrow \infty} s\left(\mathcal{G}_{2}\left[D^{2 \alpha} g(t)\right](s)+2 \alpha s^{2 \alpha-1} \mathcal{G}_{1}[g(t)](s)-2 \alpha s^{2 \alpha-1} g(0)-\alpha s^{\alpha-1} D^{\alpha} g(0)\right. \\
& \left.-\alpha s^{\alpha-1} D^{\alpha} g(0)\right) \\
& =\lim _{s \rightarrow \infty} s\left(\mathcal{G}_{2}\left[D^{2 \alpha} g(t)\right](s)\right) \\
& +\lim _{s \rightarrow \infty} 2 \alpha\left(s^{2 \alpha} \mathcal{G}_{1}[g(t)](s)-s^{2 \alpha} g(0)-s^{\alpha} D^{\alpha} g(0)\right) .
\end{aligned}
$$

Now, Lemma 2, part (i) with $n=2$ implies that

$$
h_{2}=\lim _{s \rightarrow \infty} s\left(\mathcal{G}_{2}\left[D^{2 \alpha} g(t)\right](s)\right)+2 \alpha \lim _{s \rightarrow \infty} \mathcal{G}_{1}\left[D^{2 \alpha} g(t)\right](s) \text {. }
$$


Use Corollary 1 and Property (3) to obtain

$$
h_{2}=(2 \alpha+1) D^{2 \alpha} g(0) \text {. }
$$

By similar arguments, we can predict the obvious pattern of $h_{n}$ as follows:

$$
h_{n}=(n \alpha+1) D^{n \alpha} g(0) \text {. }
$$

\section{Remark 1.}

i. If the ARA transform of order two for the function $g(t)$ has the series representation

$$
\mathcal{G}_{2}[g(t)](s)=\sum_{n=0}^{\infty} \frac{h_{n}}{s^{n \alpha+1}}
$$

then the $k^{\text {th }}$ truncated series of (9) is defined as follows:

$$
\mathcal{G}_{2}[g(t)]_{k}(s)=\sum_{n=0}^{k} \frac{h_{n}}{s^{n \alpha+1}} .
$$

The following two results come directly from Property (6) and Property (8), respectively.

ii. If the ARA transform of order two for the function $g(t)$ has the series representation

$$
\mathcal{G}_{2}[g(t)](s)=\sum_{n=0}^{\infty} \frac{h_{n}}{s^{n \alpha+1}},
$$

then the ARA transform of order one for the function $g(t)$ has the series representation

$$
\mathcal{G}_{1}[g(t)](s)=\sum_{n=0}^{\infty} \frac{h_{n}}{(n \alpha+1) s^{n \alpha}},
$$

and the $k^{\text {th }}$ truncated series is defined as follows:

$$
\mathcal{G}_{1}[g(t)]_{k}(s)=\sum_{n=0}^{k} \frac{h_{n}}{(n \alpha+1) s^{n \alpha}} .
$$

iii. The inverse of the ARA transform of order two for the fractional power series given in Theorem 4 is

$$
g(t)=\mathcal{G}_{2}^{-1}\left[\sum_{n=0}^{\infty} \frac{h_{n}}{s^{n \alpha+1}}\right](t)=\sum_{n=0}^{\infty} \frac{D^{n \alpha} g(0)}{\Gamma(n \alpha+1)} t^{n \alpha}
$$

Based on the relation between $\mathcal{G}_{2}[g(t)](s)$ and $\mathcal{G}_{1}[g(t)](s)$ given in Property (6) and the properties of Taylor's series, the following theorem includes the convergence conditions of the expansion represented in Theorem 4 for $\mathcal{G}_{2}[g(t)](s)$.

Theorem 5. Let $g(t)$ be a continuous function on every finite interval $[0, \beta]$ in which the ARA transform exists. Assume that $\mathcal{G}_{1}[g(t)](s)$ has the following series representation:

$$
\mathcal{G}_{1}[g(t)](s)=\sum_{n=0}^{\infty} \frac{C_{n}}{s^{n \alpha}}
$$

where

$$
C_{n}=D^{n \alpha} g(0)
$$


If $\left|\mathcal{G}_{1}\left[D^{(n+1) \alpha} g(t)\right](s)\right| \leq M$ on $0<s \leq d$, then the remainder $R_{n}(s)$ satisfies the following inequality:

$$
\left|R_{n}(s)\right| \leq \frac{M}{s^{(n+1) \alpha}}, 0<s \leq d .
$$

Proof Theorem 5. Suppose that $\mathcal{G}_{1}\left[D^{k \alpha} g(t)\right](s)$ exists on $0<s \leq d$ for $k=0, \ldots, n$, from the definition of the reminder,

$$
R_{n}(s)=\mathcal{G}_{1}[g(t)](s)-\sum_{k=0}^{n} \frac{D^{k \alpha} g(0)}{s^{k \alpha}} .
$$

By multiplying both sides of Equation (11) by $s^{(n+1) \alpha}$ and using part (i) of Lemma 2, we obtain

$$
s^{(n+1) \alpha} R_{n}(s)=s^{(n+1) \alpha} \mathcal{G}_{1}[g(t)](s)-\sum_{k=0}^{n} \frac{D^{k \alpha} g(0)}{s^{k \alpha}} s^{(n+1) \alpha}=\mathcal{G}_{1}\left[D^{(n+1) \alpha} g(t)\right](s) .
$$

Hence,

$$
\left|s^{(n+1) \alpha} R_{n}(s)\right|=\left|\mathcal{G}_{1}\left[D^{(n+1) \alpha} g(t)\right](s)\right| \leq M .
$$

It follows that

$$
\left|R_{n}(s)\right| \leq \frac{M}{s^{(n+1) \alpha}}, 0<s \leq d .
$$

\section{Constructing the ARA-RPS Solution of the Fractional Neutral Pantograph Equation}

In this section, we apply the ARA-RPSM to establish the solution of the FNPEs. The procedure of the presented method can be summarized as follows:

Step (1): Apply the ARA transform of order two to the given functional equation.

Step (2): Use the series expansion given in Theorem 4 to represent the solution of the new functional equation in the new space. To determine the coefficients of this expansion, we use similar arguments to the residual power series with a new analysis and new computations.

Step (3): Apply the inverse ARA transform of order two on the solution obtained in Step (2), and so, we obtain a solution in the original space.

To construct an analytical solution of Equation (1), we apply the ARA transform of order two on both sides of Equation (1)

$$
\begin{aligned}
& \mathcal{G}_{2}\left[D^{\alpha} g(t)\right](s)=a \mathcal{G}_{2}[g(\beta t)](s)+b \mathcal{G}_{2}\left[D^{\alpha} g(\gamma t)\right](s)+c \mathcal{G}_{2}[g(\lambda t)](s) \\
& +\mathcal{G}_{2}[f(t)](s) .
\end{aligned}
$$

By using part (ii) of Lemma 2 and the initial condition (2), we obtain

$$
\begin{aligned}
& s^{\alpha} \mathcal{G}_{2}[g(t)](s)-\alpha s^{\alpha-1} \mathcal{G}_{1}[g(t)](s)+(\alpha-1) s^{\alpha-1} g_{0}=\frac{a}{\beta} \mathcal{G}_{2}[g(t)]\left(\frac{s}{\beta}\right)+ \\
& b\left(\frac{s^{\alpha}}{\gamma} \mathcal{G}_{2}[g(t)]\left(\frac{s}{\gamma}\right)-\alpha s^{\alpha-1} \mathcal{G}_{1}[g(t)]\left(\frac{s}{\gamma}\right)+(\alpha-1) s^{\alpha-1} g_{0}\right)+\frac{c}{\lambda} \mathcal{G}_{2}[g(t)]\left(\frac{s}{\lambda}\right)+ \\
& \mathcal{G}_{2}[f(t)](s) .
\end{aligned}
$$

Thus, the so-called ARA-FNPE in the new space can be written as

$$
\begin{aligned}
& \mathcal{G}_{2}[g(t)](s)=\frac{\alpha}{s} \mathcal{G}_{1}[g(t)](s)-\frac{(\alpha-1)}{s} g_{0}+\frac{a}{\beta s^{\alpha}} \mathcal{G}_{2}[g(t)]\left(\frac{s}{\beta}\right)+\frac{b}{\gamma} \mathcal{G}_{2}[g(t)]\left(\frac{s}{\gamma}\right) \\
& -\frac{b \alpha}{s} \mathcal{G}_{1}[g(t)]\left(\frac{s}{\gamma}\right)+\frac{b(\alpha-1)}{s} g_{0}+\frac{c}{\lambda s^{\alpha}} \mathcal{G}_{2}[g(t)]\left(\frac{s}{\lambda}\right) \\
& +\frac{1}{s^{\alpha}} \mathcal{G}_{2}[f(t)](s) .
\end{aligned}
$$


In the second step, according to Theorem 4 and part (ii) of Remark 1, we assume that $\mathcal{G}_{1}[g(t)](s)$ and $\mathcal{G}_{2}[g(t)](s)$ in Equation (13) have the following representations:

$$
\begin{aligned}
& \mathcal{G}_{1}[g(t)](s)=\sum_{n=0}^{\infty} \frac{h_{n}}{(n \alpha+1) s^{n \alpha}}, \\
& \mathcal{G}_{2}[g(t)](s)=\sum_{n=0}^{\infty} \frac{h_{n}}{s^{n \alpha+1}}, s>0 .
\end{aligned}
$$

Consider the $k^{\text {th }}$ truncated series of Equations (14) and (15), respectively,

$$
\begin{gathered}
\mathcal{G}_{1}[g(t)]_{k}(s)=\sum_{n=0}^{k} \frac{h_{n}}{(n \alpha+1) s^{n \alpha}}, \\
\mathcal{G}_{2}[g(t)]_{k}(s)=\sum_{n=0}^{k} \frac{h_{n}}{s^{n \alpha+1}} .
\end{gathered}
$$

By multiplying both sides of Equation (17) by $s$, then taking the limit as $s \rightarrow \infty$, we obtain

$$
\lim _{s \rightarrow \infty} s \mathcal{G}_{2}[g(t)]_{k}(s)=h_{0}+\lim _{s \rightarrow \infty} \sum_{n=1}^{k} \frac{h_{n}}{s^{n \alpha}} .
$$

Corollary 1 and Equation (2) yield that

$$
h_{0}=g_{0},
$$

so, Equations (16) and (17) can be written respectively as

$$
\begin{aligned}
& \mathcal{G}_{1}[g(t)]_{k}(s)=g_{0}+\sum_{n=1}^{k} \frac{h_{n}}{(n \alpha+1) s^{n \alpha}}, \\
& \mathcal{G}_{2}[g(t)]_{k}(s)=\frac{g_{0}}{s}+\sum_{n=1}^{k} \frac{h_{n}}{s^{n \alpha+1}}, s>0 .
\end{aligned}
$$

Now, we define the so-called ARA residual function for Equation (13)

$$
\begin{aligned}
& \mathcal{G}_{2} \operatorname{Res}(s)=\mathcal{G}_{2}[g(t)](s)-\frac{\alpha}{s} \mathcal{G}_{1}[g(t)](s)+\frac{(\alpha-1)}{s} g_{0}-\frac{a}{\beta s^{\alpha}} \mathcal{G}_{2}[g(t)]\left(\frac{s}{\beta}\right) \\
& -\frac{b}{\gamma} \mathcal{G}_{2}[g(t)]\left(\frac{s}{\gamma}\right)+\frac{b \alpha}{s} \mathcal{G}_{1}[g(t)]\left(\frac{s}{\gamma}\right)-\frac{b(\alpha-1)}{s} g_{0} \\
& -\frac{c}{\lambda s^{\alpha}} \mathcal{G}_{2}[g(t)]\left(\frac{s}{\lambda}\right)-\frac{1}{s^{\alpha}} \mathcal{G}_{2}[f(t)](s),
\end{aligned}
$$

and the $k^{\text {th }}$ ARA residual function is

$$
\begin{aligned}
& \mathcal{G}_{2} \operatorname{Res}_{k}(s)=\mathcal{G}_{2}[g(t)]_{k}(s)-\frac{\alpha}{s} \mathcal{G}_{1}[g(t)]_{k}(s)+\frac{(\alpha-1)}{s} g_{0}-\frac{a}{\beta s^{\alpha}} \mathcal{G}_{2}[g(t)]_{k}\left(\frac{s}{\beta}\right) \\
& -\frac{b}{\gamma} \mathcal{G}_{2}[g(t)]_{k}\left(\frac{s}{\gamma}\right)+\frac{b \alpha}{s} \mathcal{G}_{1}[g(t)]_{k}\left(\frac{s}{\gamma}\right)-\frac{b(\alpha-1)}{s} g_{0} \\
& -\frac{c}{\lambda s^{\alpha}} \mathcal{G}_{2}[g(t)]_{k}\left(\frac{s}{\lambda}\right)-\frac{1}{s^{\alpha}} \mathcal{G}_{2}[f(t)](s) .
\end{aligned}
$$

It is clear that $\mathcal{G}_{2} \operatorname{Res}(s)=0$, and $\lim _{k \rightarrow \infty} \mathcal{G}_{2} \operatorname{Res} s_{k}(s)=\mathcal{G}_{2} \operatorname{Res}(s)$ for each $s>0$.

Since $\lim _{s \rightarrow \infty} s \mathcal{G}_{2} \operatorname{Res}(s)=0$, then $\lim _{s \rightarrow \infty} s \mathcal{G}_{2} \operatorname{Res}_{k}(s)=0$, and so

$$
\lim _{s \rightarrow \infty} s^{k \alpha+1} \mathcal{G}_{2} \operatorname{Res}(s)=\lim _{s \rightarrow \infty} s^{k \alpha+1} \mathcal{G}_{2} \operatorname{Res}_{k}(s)=0,0<\alpha \leq 1, k=1,2,3, \ldots
$$


In order to find the first unknown coefficient $h_{1}$ in Equation (18) and Equation (19), we substitute the first truncated series of $\mathcal{G}_{1}[g(t)](s)$ and $\mathcal{G}_{2}[g(t)](s)$ into the first ARA residual function $\mathcal{G}_{2} \operatorname{Res}_{1}(s)$ to obtain

$$
\begin{aligned}
& \mathcal{G}_{2} \operatorname{Res}_{1}(s)=\mathcal{G}_{2}[g(t)]_{1}(s)-\frac{\alpha}{s} \mathcal{G}_{1}[g(t)]_{1}(s)+\frac{(\alpha-1)}{s} g_{0}-\frac{a}{\beta s^{\alpha}} \mathcal{G}_{2}[g(t)]_{1}\left(\frac{s}{\beta}\right) \\
& -\frac{b}{\gamma} \mathcal{G}_{2}[g(t)]_{1}\left(\frac{s}{\gamma}\right)+\frac{b \alpha}{s} \mathcal{G}_{1}[g(t)]_{1}\left(\frac{s}{\gamma}\right)-\frac{b(\alpha-1)}{s} g_{0} \\
& -\frac{c}{\lambda s^{\alpha}} \mathcal{G}_{2}[g(t)]_{1}\left(\frac{s}{\lambda}\right)-\frac{1}{s^{\alpha}} \mathcal{G}_{2}[f(t)](s) .
\end{aligned}
$$

Substituting $\mathcal{G}_{1}[g(t)]_{1}(s)=g_{0}+\frac{h_{1}}{(\alpha+1) s^{\alpha}}$ and $\mathcal{G}_{2}[g(t)]_{1}(s)=\frac{g_{0}}{s}+\frac{h_{1}}{s^{\alpha+1}}$ in (21), we have

$$
\begin{aligned}
& \mathcal{G}_{2} \operatorname{Res}_{1}(s)=\frac{g_{0}}{s}+\frac{h_{1}}{s^{\alpha+1}}-\frac{\alpha}{s}\left(g_{0}+\frac{h_{1}}{(\alpha+1) s^{\alpha}}\right)+\frac{(\alpha-1)}{s} g_{0} \\
& -\frac{a}{\beta s^{\alpha}}\left(\frac{\beta}{s} g_{0}+\frac{\beta^{\alpha+1} h_{1}}{s^{\alpha+1}}\right)-\frac{b}{\gamma}\left(\frac{\gamma}{s} g_{0}+\frac{\gamma^{\alpha+1} h_{1}}{s^{\alpha+1}}\right) \\
& +\frac{b \alpha}{s}\left(g_{0}+\frac{\gamma^{\alpha} h_{1}}{(\alpha+1) s^{\alpha}}\right)-\frac{b(\alpha-1)}{s} g_{0}-\frac{c}{\lambda s^{\alpha}}\left(\frac{\lambda g_{0}}{s}+\frac{\lambda^{\alpha+1} h_{1}}{s^{\alpha+1}}\right) \\
& -\frac{1}{s^{\alpha}} \mathcal{G}_{2}[f(t)](s) \\
& =(-a-c) \frac{g_{0}}{s^{\alpha+1}}+\frac{h_{1}}{s^{\alpha+1}}\left(\frac{1-b \gamma^{\alpha}}{\alpha+1}\right)-\frac{h_{1}}{s^{2 \alpha+1}}\left(a \beta^{\alpha}+c \lambda^{\alpha}\right) \\
& -\frac{1}{s^{\alpha}} \mathcal{G}_{2}[f(t)](s) .
\end{aligned}
$$

By multiplying both sides of (22) by $s^{\alpha+1}$ and taking the limit as $s \rightarrow \infty$, we obtain

$$
\lim _{s \rightarrow \infty} s^{\alpha+1} \mathcal{G}_{2} \operatorname{Res}_{1}(s)=(-a-c) g_{0}+h_{1}\left(\frac{1-b \gamma^{\alpha}}{\alpha+1}\right)-\lim _{s \rightarrow \infty} \mathcal{G}_{2}[f(t)](s)
$$

Corollary 1 and the facts in Equation (20) yield

$$
-(a+c) g_{0}+h_{1}\left(\frac{1-b \gamma^{\alpha}}{\alpha+1}\right)-f(0)=0
$$

and so

$$
h_{1}=\frac{\alpha+1}{1-b \gamma^{\alpha}}\left[(a+c) g_{0}+f(0)\right] .
$$

Thus, the second truncated series of $\mathcal{G}_{1}[g(t)](s)$ and $\mathcal{G}_{2}[g(t)](s)$ are

$$
\mathcal{G}_{1}[g(t)]_{2}(s)=g_{0}+\frac{h_{1}}{(\alpha+1) s^{\alpha}}+\frac{h_{2}}{(2 \alpha+1) s^{2 \alpha}}
$$

and

$$
\mathcal{G}_{2}[g(t)]_{2}(s)=\frac{g_{0}}{s}+\frac{h_{1}}{s^{\alpha+1}}+\frac{h_{2}}{s^{2 \alpha+1}} .
$$

To find $h_{2}$, we substitute $\mathcal{G}_{1}[g(t)]_{2}(s)$ and $\mathcal{G}_{2}[g(t)]_{2}(s)$ in the second ARA residual function $\mathcal{G}_{2} \operatorname{Res}_{2}(s)$, to obtain

$$
\begin{aligned}
& \mathcal{G}_{2} \operatorname{Res}_{2}(s)=\frac{g_{0}}{s}+\frac{h_{1}}{s^{\alpha+1}}+\frac{h_{2}}{s^{2 \alpha+1}}-\frac{\alpha}{s}\left(g_{0}+\frac{h_{1}}{(\alpha+1) s^{\alpha}}+\frac{h_{2}}{(2 \alpha+1) s^{2 \alpha}}\right)+\frac{(\alpha-1)}{s} g_{0} \\
& -\frac{a}{\beta s^{\alpha}}\left(\frac{\beta g_{0}}{s}+\frac{\beta^{\alpha+1} h_{1}}{s^{\alpha+1}}+\frac{\beta^{2 \alpha+1} h_{2}}{s^{2 \alpha+1}}\right)-\frac{b}{\gamma}\left(\frac{\gamma g_{0}}{s}+\frac{\gamma^{\alpha+1} h_{1}}{s^{\alpha+1}}+\frac{\gamma^{2 \alpha+1} h_{2}}{s^{2 \alpha+1}}\right) \\
& +\frac{b \alpha}{s}\left(g_{0}+\frac{\gamma^{\alpha} h_{1}}{(\alpha+1) s^{\alpha}}+\frac{\gamma^{2 \alpha} h_{2}}{(2 \alpha+1) s^{2 \alpha}}\right)-\frac{b(\alpha-1)}{s} g_{0} \\
& -\frac{c}{\lambda s^{\alpha}}\left(\frac{\lambda g_{0}}{s}+\frac{\lambda^{\alpha+1} h_{1}}{s^{\alpha+1}}+\frac{\lambda^{2 \alpha+1} h_{2}}{s^{2 \alpha+1}}\right)-\frac{1}{s^{\alpha}} \mathcal{G}_{2}[f(t)](s),
\end{aligned}
$$


after simple computations, $\mathcal{G}_{2} \operatorname{Res}_{2}(s)$ can be written as

$$
\begin{aligned}
& \mathcal{G}_{2} \operatorname{Res}_{2}(s)=\left(-\frac{a+c}{s^{\alpha+1}}\right) g_{0}+\frac{h_{1}}{s^{\alpha+1}}\left(\frac{1-b \gamma^{\alpha}}{\alpha+1}\right) \\
& +\frac{1}{s^{2 \alpha+1}}\left(h_{2} \frac{(\alpha+1)\left(1-b \gamma^{2 \alpha}\right)}{2 \alpha+1}-h_{1}\left(a \beta^{\alpha}+c \lambda^{\alpha}\right)\right) \\
& -\frac{h_{2}}{s^{3 \alpha+1}}\left(a \beta^{2 \alpha}+c \lambda^{2 \alpha}\right)-\frac{1}{s^{\alpha}} \mathcal{G}_{2}[f(t)](s) .
\end{aligned}
$$

By multiplying both sides of Equation (24) by $s^{2 \alpha+1}$ and taking the limit as $s \rightarrow \infty$, we have:

$$
\begin{aligned}
& \lim _{s \rightarrow \infty} s^{2 \alpha+1} \mathcal{G}_{2} \operatorname{Res}_{2}(s) \\
& =-\lim _{s \rightarrow \infty} s^{\alpha}(a+c) g_{0}+\lim _{s \rightarrow \infty} s^{\alpha} h_{1}\left(\frac{1-b \gamma^{\alpha}}{\alpha+1}\right)+h_{2} \frac{(\alpha+1)\left(1-b \gamma^{2 \alpha}\right)}{2 \alpha+1} \\
& -h_{1}\left(a \beta^{\alpha}+c \lambda^{\alpha}\right)-\lim _{s \rightarrow \infty} s^{\alpha+1} \mathcal{G}_{2}[f(t)](s) .
\end{aligned}
$$

By using the facts in Equation (20), $h_{2}$ has the following form:

$$
\begin{aligned}
& h_{2}=\frac{2 \alpha+1}{(\alpha+1)\left(1-b \gamma^{2 \alpha}\right)}\left[h_{1}\left(a \beta^{\alpha}+c \lambda^{\alpha}\right)+\lim _{s \rightarrow \infty} s^{\alpha+1} \mathcal{G}_{2}[f(t)](s)\right. \\
& \left.+\lim _{s \rightarrow \infty} s^{\alpha}(a+c) g_{0}-\lim _{s \rightarrow \infty} s^{\alpha} h_{1}\left(\frac{1-b \gamma^{\alpha}}{\alpha+1}\right)\right] .
\end{aligned}
$$

Equation (23) implies that

$$
\lim _{s \rightarrow \infty}\left(s^{\alpha}(a+c) g_{0}-s^{\alpha} h_{1}\left(\frac{1-b \gamma^{\alpha}}{\alpha+1}\right)\right)=\lim _{s \rightarrow \infty}\left(-s^{\alpha} f(0)\right) .
$$

So, Equation (25) is equivalent to

$$
h_{2}=\frac{2 \alpha+1}{(\alpha+1)\left(1-b \gamma^{2 \alpha}\right)}\left[h_{1}\left(a \beta^{\alpha}+c \lambda^{\alpha}\right)+\lim _{s \rightarrow \infty}\left(s^{\alpha+1} \mathcal{G}_{2}[f(t)](s)-s^{\alpha} f(0)\right)\right] .
$$

According to part (ii) of Lemma 2, $h_{2}$ becomes

$$
\begin{aligned}
& h_{2}=\frac{2 \alpha+1}{(\alpha+1)\left(1-b \gamma^{2 \alpha}\right)}\left[h_{1}\left(a \beta^{\alpha}+c \lambda^{\alpha}\right)+\lim _{s \rightarrow \infty} s\left(\mathcal{G}_{2}\left[D^{\alpha} f(t)\right](s)\right)\right. \\
& \left.+\alpha \lim _{s \rightarrow \infty} s^{\alpha} \mathcal{G}_{1}[f(t)](s)-s^{\alpha} f(0)\right] .
\end{aligned}
$$

Again, part (i) of Lemma 2 with $n=1$ will give

$$
\begin{aligned}
& h_{2}=\frac{2 \alpha+1}{(\alpha+1)\left(1-b \gamma^{2 \alpha}\right)}\left[h_{1}\left(a \beta^{\alpha}+c \lambda^{\alpha}\right)+\lim _{s \rightarrow \infty} s\left(\mathcal{G}_{2}\left[D^{\alpha} f(t)\right](s)\right)\right. \\
& \left.+\alpha \lim _{s \rightarrow \infty} \mathcal{G}_{1}\left[D^{\alpha} f(t)\right](s)\right] .
\end{aligned}
$$

Then utilizing Corollary 1 and Property (3) to obtain

$$
h_{2}=\frac{2 \alpha+1}{(\alpha+1)\left(1-b \gamma^{2 \alpha}\right)}\left[h_{1}\left(a \beta^{\alpha}+c \lambda^{\alpha}\right)+(1+\alpha) D^{\alpha} f(0)\right] .
$$


For $k=3$, the third ARA residual function $\mathcal{G}_{2} \operatorname{Res}_{3}(s)$ can be written as

$$
\begin{gathered}
\mathcal{G}_{2} \operatorname{Res}_{3}(s)=\frac{g_{0}}{s}+\frac{h_{1}}{s^{\alpha+1}}+\frac{h_{2}}{s^{2 \alpha+1}}+\frac{h_{3}}{s^{3 \alpha+1}} \\
-\frac{\alpha}{s}\left(g_{0}+\frac{h_{1}}{(\alpha+1) s^{\alpha}}+\frac{h_{2}}{(2 \alpha+1) s^{2 \alpha}}+\frac{h_{3}}{(3 \alpha+1) s^{3 \alpha}}\right)+\frac{(\alpha-1)}{s} g_{0} \\
-\frac{a}{\beta s^{\alpha}}\left(\frac{\beta g_{0}}{s}+\frac{\beta^{\alpha+1} h_{1}}{s^{\alpha+1}}+\frac{\beta^{2 \alpha+1} h_{2}}{s^{2 \alpha+1}}+\frac{\beta^{3 \alpha+1} h_{3}}{s^{3 \alpha+1}}\right) \\
-\frac{b}{\gamma}\left(\frac{\gamma g_{0}}{s}+\frac{\gamma^{\alpha+1} h_{1}}{s^{\alpha+1}}+\frac{\gamma^{2 \alpha+1} h_{2}}{s^{2 \alpha+1}}+\frac{\gamma^{3 \alpha+1} h_{3}}{s^{3 \alpha+1}}\right) \\
+\frac{b \alpha}{s}\left(g_{0}+\frac{\gamma^{\alpha} h_{1}}{(\alpha+1) s^{\alpha}}+\frac{\gamma^{2 \alpha} h_{2}}{(2 \alpha+1) s^{2 \alpha}}+\frac{\gamma^{3 \alpha} h_{3}}{(3 \alpha+1) s^{3 \alpha}}\right) \\
-\frac{b(\alpha-1)}{s} g_{0}-\frac{c}{\lambda s^{\alpha}}\left(\frac{\lambda g_{0}}{s}+\frac{\lambda^{\alpha+1} h_{1}}{s^{\alpha+1}}+\frac{\lambda^{2 \alpha+1} h_{2}}{s^{2 \alpha+1}}+\frac{\lambda^{3 \alpha+1} h_{3}}{s^{3 \alpha+1}}\right) \\
-\frac{1}{s^{\alpha}} \mathcal{G}_{2}[f(t)](s) . \\
\mathcal{G}_{2} \operatorname{Res}_{3}(s)=\frac{f(0)}{s^{\alpha+1}}-\frac{h_{1}}{s^{2 \alpha+1}}\left(a \beta^{\alpha}+c \lambda^{\alpha}\right)+\frac{h_{2}}{s^{2 \alpha+1}}\left(\frac{(1+\alpha)\left(1-b \gamma^{2 \alpha}\right)}{2 \alpha+1}\right) \\
-\frac{h_{2}}{s^{3 \alpha+1}}\left(a \beta^{2 \alpha}+c \lambda^{2 \alpha}\right)+\frac{h_{3}}{s^{3 \alpha+1}}\left(\frac{(1+2 \alpha)\left(1-b \gamma^{3 \alpha}\right)}{3 \alpha+1}\right) \\
-\frac{h_{3}}{s^{4 \alpha+1}}\left(a \beta^{3 \alpha}+c \lambda^{3 \alpha}\right)-\frac{1}{s^{\alpha}} \mathcal{G}_{2}[f(t)](s) .
\end{gathered}
$$

By multiplying both sides of Equation (26) by $s^{3 \alpha+1}$ and taking the limit as $s \rightarrow \infty$, we obtain

$$
\begin{aligned}
& \lim _{s \rightarrow \infty} s^{3 \alpha+1} \mathcal{G}_{2} \operatorname{Res}_{3}(s) \\
& =\lim _{s \rightarrow \infty} s^{2 \alpha} f(0)-\lim _{s \rightarrow \infty} s^{\alpha} h_{1}\left(a \beta^{\alpha}+c \lambda^{\alpha}\right) \\
& +\lim _{s \rightarrow \infty} s^{\alpha} h_{2}\left(\frac{(1+\alpha)\left(1-b \gamma^{2 \alpha}\right)}{2 \alpha+1}\right)-h_{2}\left(a \beta^{2 \alpha}+c \lambda^{2 \alpha}\right) \\
& +h_{3}\left(\frac{(1+2 \alpha)\left(1-b \gamma^{3 \alpha}\right)}{3 \alpha+1}\right)-\lim _{s \rightarrow \infty} \frac{h_{3}}{s^{\alpha}}\left(a \beta^{3 \alpha}+c \lambda^{3 \alpha}\right) \\
& -\lim _{s \rightarrow \infty} s^{2 \alpha+1} \mathcal{G}_{2}[f(t)](s) .
\end{aligned}
$$

The facts in Equation (20) and Lemma 2 lead after simple computations to the following form of $h_{3}$ :

$$
h_{3}=\frac{3 \alpha+1}{(1+2 \alpha)\left(1-b \gamma^{3 \alpha}\right)}\left(h_{2}\left(a \beta^{2 \alpha}+c \lambda^{2 \alpha}\right)+(1+2 \alpha) D^{2 \alpha} f(0)\right)
$$

The reader can predict an obvious pattern for the unknown coefficients of the series in Equation (14). Hence, the formula of $h_{n}$ can be written recurrently as

$$
h_{n}= \begin{cases}\frac{n \alpha+1}{((n-1) \alpha+1)\left(1-b \gamma^{n \alpha}\right)}\left[h_{n-1}\left(a \beta^{(n-1) \alpha}+c \lambda^{(n-1) \alpha}\right)\right. & , n=1,2, \cdots \\ \left.+((n-1) \alpha+1) D^{(n-1) \alpha} f(0)\right] & \\ g_{0} & , n=0 .\end{cases}
$$


Now, we are able to write the $\mathrm{k}^{\text {th }}$ approximate solution of Equation (12) as follows:

$$
\begin{aligned}
& \mathcal{G}_{2}[g(t)]_{k}(s)=\frac{g_{0}}{s}+\frac{1}{s^{\alpha+1}}\left(\frac{\alpha+1}{1-b \gamma^{\alpha}}\left[(a+c) g_{0}+f(0)\right]\right) \\
& +\frac{1}{s^{2 \alpha+1}}\left(\frac { 2 \alpha + 1 } { ( \alpha + 1 ) ( 1 - b \gamma ^ { 2 \alpha } ) } \left[\left(a \beta^{\alpha}+c \lambda^{\alpha}\right) h_{1}\right.\right. \\
& \left.\left.+(\alpha+1) D^{\alpha} f(0)\right]\right) \\
& +\frac{1}{s^{3 \alpha+1}}\left(\frac { 3 \alpha + 1 } { ( 2 \alpha + 1 ) ( 1 - b \gamma ^ { 3 \alpha } ) } \left[\left(a \beta^{2 \alpha}+c \lambda^{2 \alpha}\right) h_{2}\right.\right. \\
& \left.\left.+(2 \alpha+1) D^{2 \alpha} f(0)\right]\right)+\ldots \\
& +\frac{1}{s^{k \alpha+1}}\left(\frac { k \alpha + 1 } { ( ( k - 1 ) \alpha + 1 ) ( 1 - b \gamma ^ { k \alpha } ) } \left[h _ { k - 1 } \left(a \beta^{(k-1) \alpha}\right.\right.\right. \\
& \left.\left.\left.+c \lambda^{(k-1) \alpha}\right)+((k-1) \alpha+1) D^{(k-1) \alpha} f(0)\right]\right) .
\end{aligned}
$$

Finally, the last step of the ARA-RPSM is applying the inverse ARA transform of order two to both sides of Equation (28) to obtain the solution of the FNPE in Equations (1) and (2). Thus, the $\mathrm{k}^{\text {th }}$ approximate solution of the FNPE in the original space is given by

$$
\begin{aligned}
& g_{k}(t) \\
& =g_{0}+\frac{t^{\alpha}}{\Gamma(\alpha+1)}\left(\frac{(a+c) g_{0}+f(0)}{1-b \gamma^{\alpha}}\right) \\
& +\frac{t^{2 \alpha}}{\Gamma(2 \alpha+1)}\left(\frac{\left(a \beta^{\alpha}+c \lambda^{\alpha}\right) h_{1}+(\alpha+1) D^{\alpha} f(0)}{(\alpha+1)\left(1-b \gamma^{2 \alpha}\right)}\right) \\
& +\frac{t^{3 \alpha}}{\Gamma(3 \alpha+1)}\left(\frac{\left(a \beta^{2 \alpha}+c \lambda^{2 \alpha}\right) h_{2}+(2 \alpha+1) D^{2 \alpha} f(0)}{(2 \alpha+1)\left(1-b \gamma^{3 \alpha}\right)}\right)+\ldots \\
& +\frac{t^{k \alpha}}{\Gamma(k \alpha+1)}\left(\frac{\left(a \beta^{(k-1) \alpha}+c \lambda^{(k-1) \alpha}\right) h_{k-1}+((k-1) \alpha+1) D^{(k-1) \alpha} f(0)}{((k-1) \alpha+1)\left(1-b \gamma^{k \alpha}\right)}\right) \\
& g_{k}(t) \\
& =g(0)+\sum_{i=1}^{k} \frac{t^{i \alpha}}{\Gamma(i \alpha+1)}\left(\frac{\left(a \beta^{(i-1) \alpha}+c \lambda^{(i-1) \alpha}\right) h_{(i-1)}+((i-1) \alpha+1) D^{(i-1) \alpha} f(0)}{((i-1) \alpha+1)\left(1-b \gamma^{i \alpha}\right)}\right)
\end{aligned}
$$

\section{Some Numerical Examples}

Through this section, we introduce two interesting examples to illustrate the simplicity, efficiency, and accuracy of the ARA-RPSM.

In this section, it is good to mention that we use Mathematica 12 software to obtain the numerical results.

Example 1. Consider the following linear NFPE:

$$
D^{1 / 2} g(t)=-g(t)+g\left(\frac{t}{2}\right)+\frac{7}{8} t^{3}+\frac{16}{5 \Gamma(0.5)} t^{\frac{5}{2}}, t \geq 0,0<\alpha \leq 1,
$$

with the initial condition

$$
g(0)=0 .
$$

Solution. To apply the proposed method, we compare Equations (29) and (30) with Equations (1) and (22) to obtain

$$
\begin{aligned}
a=-1, \beta=1, b=0, \gamma=0, c & =1, \lambda=\frac{1}{2}, f(t)=\frac{7}{8} t^{3}+\frac{16}{5 \Gamma(0.5)} t^{5 / 2} \\
g(0) & =g_{0}=0
\end{aligned}
$$


Thus, according to the formula of $h_{n}$ obtained in Equation (27), we have

$$
h_{n}= \begin{cases}(n \alpha+1)\left(\frac{h_{n-1}\left(-1+2^{-(n-1) \alpha}\right)}{((n-1) \alpha+1)}+D^{(n-1) \alpha} f(0)\right) & , n=1,2, \cdots \\ 0 & , n=0 .\end{cases}
$$

By putting $\alpha=1 / 2$ in Equation (31), with simple computations, we obtain

$$
h_{n}=\frac{n+2}{n+1} h_{n-1}\left(-1+2^{\frac{1-n}{2}}\right)+\left(\frac{n}{2}+1\right)\left(D^{\frac{1}{2}}\right)^{n-1} f(0)
$$

To find out $h_{n}$, we should determine the value of $D^{(n-1) \alpha} f(0)$ for $n=1,2, \ldots$, in Equation (32).

$$
\begin{array}{ll}
D^{1 / 2} f(t)=\frac{14 t^{5 / 2}}{5 \sqrt{\pi}}+3 t^{2} & \left(D^{\frac{1}{2}}\right)^{4} f(t)=\frac{21 t}{4}+\frac{12 \sqrt{t}}{\sqrt{\pi}} \\
\left(D^{\frac{1}{2}}\right)^{2} f(t)=\frac{21 t^{2}}{8}+\frac{8 t^{3 / 2}}{\sqrt{\pi}} & \left(D^{\frac{1}{2}}\right)^{5} f(t)=\frac{21 \sqrt{t}}{2 \sqrt{\pi}}+6 \\
\left(D^{\frac{1}{2}}\right)^{3} f(t)=\frac{7 t^{3 / 2}}{\sqrt{\pi}}+6 t & \left(D^{\frac{1}{2}}\right)^{6} f(t)=\frac{21}{4}
\end{array}
$$

According to Equation (32), the values of $h_{n}$ can be easily obtained as

$$
h_{n}= \begin{cases}24, & n=6 \\ 0, & n \neq 6\end{cases}
$$

Back to Equation (28), the approximate solution of the ARA transform of order two for the solution of Equation (29) is presented in the form

$$
\mathcal{G}_{2}[g(t)](s)=\sum_{n=0}^{\infty} \frac{h_{n}}{S^{n \alpha+1}}=\frac{h_{6}}{s^{4}}=\frac{24}{s^{4}}
$$

By applying the inverse ARA transform of order two, to both sides of Equation (33), we obtain $g(t)=t^{3}$, which is the exact solution of Equations (29) and (30) obtained in [38].

Example 2. Consider the following linear NFPE

$$
D^{\alpha} g(t)=-g(t)+\frac{1}{10} g\left(\frac{4}{5} t\right)+\frac{1}{2}\left(\frac{5}{4}\right)^{\alpha} D^{\alpha} g\left(\frac{4}{5} t\right)+\left(\frac{8 t^{\alpha}}{25}-\frac{1}{2}\right) e^{-\frac{4}{5} t^{\alpha}}+e^{-t \alpha},
$$

with the initial condition

$$
g(0)=0
$$

Solution. To apply the proposed method, we compare Equations (34) and (35) with Equations (1) and (2) to obtain

$$
\begin{aligned}
a=-1, b=\frac{1}{2}\left(\frac{5}{4}\right)^{\alpha}, c=\frac{1}{10}, \beta=1, \gamma & =\frac{4}{5}, \lambda=\frac{4}{5}, f(t)=\left(\frac{8 t^{\alpha}}{25}-\frac{1}{2}\right) e^{-\frac{4}{5} t^{\alpha}}+e^{-t \alpha} \\
g(0) & =g_{0}=0
\end{aligned}
$$


Thus, according to the formula of $h_{n}$ obtained in Equation (27), we have

$$
h_{n}= \begin{cases}\frac{n \alpha+1}{\left(1-\frac{1}{2}\left(\frac{5}{4}\right)^{\alpha}\left(\frac{4}{5}\right)^{n \alpha}\right)}\left(\frac{h_{n-1}\left(-1+\frac{1}{10}\left(\frac{4}{5}\right)^{(n-1) \alpha}\right)}{((n-1) \alpha+1)}+D^{(n-1) \alpha} f(0)\right) & , n=1,2, \ldots \\ 0 & , n=0 .\end{cases}
$$

To find out $h_{n}$, we should determine the value of $D^{(n-1) \alpha} f(0)$ for $n=1,2, \ldots$, in Equation (36). For simplicity, in calculating the terms $D^{(n-1) \alpha} f(0)$, we use the first six terms of the expansion of $f(t)$ :

$$
f(t)=\frac{1}{2}-\frac{7 t^{\alpha}}{25}+\frac{21 t^{2 \alpha}}{250}-\frac{27 t^{3 \alpha}}{1250}+\frac{437 t^{4 \alpha}}{75,000}-\frac{113 t^{5 \alpha}}{75,000},
$$

and so,

$$
\begin{aligned}
& D^{\alpha} f(t)=-\frac{7}{25} \Gamma(1+\alpha)+\frac{21 \alpha^{\alpha} \Gamma(1+2 \alpha)}{250 \Gamma(1+\alpha)}-\frac{27 t^{2 \alpha} \Gamma(1+3 \alpha)}{1250 \Gamma(1+2 \alpha)}+\frac{437 t^{3 \alpha} \Gamma(1+4 \alpha)}{75,000 \Gamma(1+3 \alpha)} \\
& -\frac{113 t^{4 \alpha} \Gamma(1+5 \alpha)}{75,000 \Gamma(1+4 \alpha)} \\
& D^{2 \alpha} f(t)=\frac{21 \Gamma(1+2 \alpha)}{250}-\frac{27 t^{\alpha} \Gamma(1+3 \alpha)}{125 \Gamma(1+\alpha)}+\frac{437 t^{2 \alpha} \Gamma(1+4 \alpha)}{75,000 \Gamma(1+2 \alpha)}-\frac{113 t^{3 \alpha} \Gamma(1+5 \alpha)}{75,000 \Gamma(1+3 \alpha)} \\
& D^{3 \alpha} f(t)=-\frac{27 \Gamma(1+3 \alpha)}{125}+\frac{437 t^{\alpha} \Gamma(1+4 \alpha)}{75,000 \Gamma(1+\alpha)}-\frac{113 t^{2 \alpha} \Gamma(1+5 \alpha)}{75,000 \Gamma(1+2 \alpha)} \\
& D^{4 \alpha} f(t)=\frac{437 \Gamma(1+4 \alpha)}{75,000}-\frac{113 t^{\alpha} \Gamma(1+5 \alpha)}{75,000 \Gamma(1+\alpha)} \\
& D^{5 \alpha} f(t)=-\frac{113 \Gamma(1+5 \alpha)}{75,000}
\end{aligned}
$$

Thus, the values of $h_{n}$ for $n=0,1,2,3,4,5,6$, can be easily obtained as:

$$
\begin{aligned}
& h_{0}=0 \\
& h_{1}=\alpha+1 \\
& h_{2}=\frac{2(5)^{\alpha}(25+7 \Gamma(1+\alpha))-5(4)^{\alpha}}{25\left(4^{\alpha}-2(5)^{\alpha}\right)}(2 \alpha+1) \\
& h_{3}=\left(\frac{\left(16^{\alpha}-2(5)^{1+2 \alpha}\right)\left(5(4)^{\alpha}-2(5)^{\alpha}(25+7 \Gamma(1+\alpha))\right)}{125\left(4^{\alpha}-2(5)^{\alpha}\right)\left(16^{\alpha}-2(25)^{\alpha}\right)}+\frac{21 \Gamma(1+2 \alpha)}{250-5^{3-2 \alpha} 16^{\alpha}}\right)(3 \alpha \\
& +1) \\
& h_{4} \\
& =\left(\frac{27 \Gamma(1+3 \alpha)}{625(64)^{\alpha}(125)^{-\alpha}-1250}\right. \\
& -\frac{\left(64^{\alpha}-10(125)^{\alpha}\right)}{5(64)^{\alpha}-10(125)^{\alpha}}\left(\frac{\left(64^{\alpha}-10(100)^{\alpha}\right)\left(-5(4)^{\alpha}(5)^{-\alpha}+50+14 \Gamma(1+\alpha)\right)}{125\left(2-4^{\alpha}(5)^{-\alpha}\right)\left(64^{\alpha}-2(100)^{\alpha}\right)}\right. \\
& \left.\left.+\frac{21 \Gamma(1+2 \alpha)}{250-5^{3-2 \alpha} 16^{\alpha}}\right)\right)(4 \alpha+1), \\
& h_{5} \\
& =\left(\frac{-437 \Gamma(1+4 \alpha)}{37,500(256)^{\alpha}(625)^{-\alpha}-75,000}\right. \\
& -\frac{(256)^{\alpha}-10(625)^{\alpha}}{5(256)^{\alpha}-10(625)^{\alpha}}\left(\frac{27 \Gamma(1+3 \alpha)}{625(64)^{\alpha}(125)^{-\alpha}-1250}\right.
\end{aligned}
$$




$$
\begin{aligned}
& -\frac{\left(64^{\alpha}-10(125)^{\alpha}\right)}{5(64)^{\alpha}-10(125)^{\alpha}}\left(\frac{\left(64^{\alpha}-10(100)^{\alpha}\right)\left(-5(4)^{\alpha}(5)^{-\alpha}+50+14 \Gamma(1+\alpha)\right)}{125\left(2-4^{\alpha}(5)^{-\alpha}\right)\left(64^{\alpha}-2(100)^{\alpha}\right)}\right. \\
& \left.\left.\left.+\frac{21 \Gamma(1+2 \alpha)}{250-5^{3-2 \alpha} 16^{\alpha}}\right)\right)\right)(5 \alpha+1), \\
& h_{6} \\
& =\left(\frac{113 \Gamma(1+5 \alpha)}{37,500(1024)^{\alpha}(3125)^{-\alpha}-75,000}\right. \\
& -\frac{(1024)^{\alpha}-10(3125)^{\alpha}}{5(1024)^{\alpha}-10(3125)^{\alpha}}\left(\frac{-437 \Gamma(1+4 \alpha)}{37,500(256)^{\alpha}(625)^{-\alpha}-75,000}\right. \\
& -\frac{(256)^{\alpha}-10(625)^{\alpha}}{5(256)^{\alpha}-10(625)^{\alpha}}\left(\frac{27 \Gamma(1+3 \alpha)}{625(64)^{\alpha}(125)^{-\alpha}-1250}\right. \\
& -\frac{\left(64^{\alpha}-10(125)^{\alpha}\right)}{5(64)^{\alpha}-10(125)^{\alpha}}\left(\frac{\left(64^{\alpha}-10(100)^{\alpha}\right)\left(-5(4)^{\alpha}(5)^{-\alpha}+50+14 \Gamma(1+\alpha)\right)}{125\left(2-4^{\alpha}(5)^{-\alpha}\right)\left(64^{\alpha}-2(100)^{\alpha}\right)}\right. \\
& \left.\left.\left.+\frac{21 \Gamma(1+2 \alpha)}{250-5^{3-2 \alpha} 16^{\alpha}}\right)\right)\right)(6 \alpha+1) .
\end{aligned}
$$

Back to Equation (28), the 6th approximate solution of the ARA transform of order two for the solution of Equation (34) is presented in the form

$$
\begin{aligned}
& \mathcal{G}_{2}[g(t)]_{6}(s)=\sum_{n=0}^{6} \frac{h_{n}}{S^{n \alpha+1}} \\
& =\frac{\alpha+1}{S^{\alpha+1}}+\frac{2 \alpha+1}{S^{2 \alpha+1}}\left(\frac{2(5)^{\alpha}(25+7 \Gamma(1+\alpha))-5(4)^{\alpha}}{25\left(4^{\alpha}-2(5)^{\alpha}\right)}\right) \\
& +\frac{3 \alpha+1}{S^{3 \alpha+1}}\left(\frac{\left(16^{\alpha}-2(5)^{1+2 \alpha}\right)\left(5(4)^{\alpha}-2(5)^{\alpha}(25+7 \Gamma(1+\alpha))\right)}{125\left(4^{\alpha}-2(5)^{\alpha}\right)\left(16^{\alpha}-2(25)^{\alpha}\right)}\right. \\
& \left.+\frac{21 \Gamma(1+2 \alpha)}{250-5^{3-2 \alpha} 16^{\alpha}}\right) \\
& +\frac{4 \alpha+1}{S^{4 \alpha+1}}\left(\frac{27 \Gamma(1+3 \alpha)}{625(64)^{\alpha}(125)^{-\alpha}-1250}\right. \\
& -\frac{\left(64^{\alpha}-10(125)^{\alpha}\right)}{5(64)^{\alpha}-10(125)^{\alpha}}\left(\frac{\left(64^{\alpha}-10(100)^{\alpha}\right)\left(-5(4)^{\alpha}(5)^{-\alpha}+50+14 \Gamma(1+\alpha)\right)}{125\left(2-4^{\alpha}(5)^{-\alpha}\right)\left(64^{\alpha}-2(100)^{\alpha}\right)}\right. \\
& \left.\left.+\frac{21 \Gamma(1+2 \alpha)}{250-5^{3-2 \alpha} 16^{\alpha}}\right)\right) \\
& +\frac{5 \alpha+1}{S^{5 \alpha+1}}\left(\frac{-437 \Gamma(1+4 \alpha)}{37,500(256)^{\alpha}(625)^{-\alpha}-75,000}\right. \\
& -\frac{(256)^{\alpha}-10(625)^{\alpha}}{5(256)^{\alpha}-10(625)^{\alpha}}\left(\frac{27 \Gamma(1+3 \alpha)}{625(64)^{\alpha}(125)^{-\alpha}-1250}\right. \\
& -\frac{\left(64^{\alpha}-10(125)^{\alpha}\right)}{5(64)^{\alpha}-10(125)^{\alpha}}\left(\frac{\left(64^{\alpha}-10(100)^{\alpha}\right)\left(-5(4)^{\alpha}(5)^{-\alpha}+50+14 \Gamma(1+\alpha)\right)}{125\left(2-4^{\alpha}(5)^{-\alpha}\right)\left(64^{\alpha}-2(100)^{\alpha}\right)}\right. \\
& \left.\left.\left.+\frac{21 \Gamma(1+2 \alpha)}{250-5^{3-2 \alpha} 16^{\alpha}}\right)\right)\right) \\
& +\frac{6 \alpha+1}{S^{6 \alpha+1}}\left(\frac{113 \Gamma(1+5 \alpha)}{37,500(1024)^{\alpha}(3125)^{-\alpha}-75,000}\right. \\
& -\frac{(1024)^{\alpha}-10(3125)^{\alpha}}{5(1024)^{\alpha}-10(3125)^{\alpha}}\left(\frac{-437 \Gamma(1+4 \alpha)}{37,500(256)^{\alpha}(625)^{-\alpha}-75,000}\right. \\
& -\frac{(256)^{\alpha}-10(625)^{\alpha}}{5(256)^{\alpha}-10(625)^{\alpha}}\left(\frac{27 \Gamma(1+3 \alpha)}{625(64)^{\alpha}(125)^{-\alpha}-1250}\right. \\
& -\frac{\left(64^{\alpha}-10(125)^{\alpha}\right)}{5(64)^{\alpha}-10(125)^{\alpha}}\left(\frac{\left(64^{\alpha}-10(100)^{\alpha}\right)\left(-5(4)^{\alpha}(5)^{-\alpha}+50+14 \Gamma(1+\alpha)\right)}{125\left(2-4^{\alpha}(5)^{-\alpha}\right)\left(64^{\alpha}-2(100)^{\alpha}\right)}\right. \\
& \left.\left.\left.\left.+\frac{21 \Gamma(1+2 \alpha)}{250-5^{3-2 \alpha} 16^{\alpha}}\right)\right)\right)\right) \text {. }
\end{aligned}
$$


Hence, by applying the inverse ARA transform of order two, on both sides of Equation (37), we obtain the 6th approximate solution of Equation (34)

$$
\begin{aligned}
& g_{6}(t) \\
& =\frac{t^{\alpha}}{\Gamma(\alpha+1)}+\frac{t^{2 \alpha}}{\Gamma(2 \alpha+1)}\left(\frac{2(5)^{\alpha}(25+7 \Gamma(1+\alpha))-5(4)^{\alpha}}{25\left(4^{\alpha}-2(5)^{\alpha}\right)}\right) \\
& +\frac{t^{3 \alpha}}{\Gamma(3 \alpha+1)}\left(\frac{\left(16^{\alpha}-2(5)^{1+2 \alpha}\right)\left(5(4)^{\alpha}-2(5)^{\alpha}(25+7 \Gamma(1+\alpha))\right)}{125\left(4^{\alpha}-2(5)^{\alpha}\right)\left(16^{\alpha}-2(25)^{\alpha}\right)}\right. \\
& \left.+\frac{21 \Gamma(1+2 \alpha)}{250-5^{3-2 \alpha} 16^{\alpha}}\right) \\
& +\frac{t^{4 \alpha}}{\Gamma(4 \alpha+1)}\left(\frac{27 \Gamma(1+3 \alpha)}{625(64)^{\alpha}(125)^{-\alpha}-1250}\right. \\
& -\frac{\left(64^{\alpha}-10(125)^{\alpha}\right)}{5(64)^{\alpha}-10(125)^{\alpha}}\left(\frac{\left(64^{\alpha}-10(100)^{\alpha}\right)\left(-5(4)^{\alpha}(5)^{-\alpha}+50+14 \Gamma(1+\alpha)\right)}{125\left(2-4^{\alpha}(5)^{-\alpha}\right)\left(64^{\alpha}-2(100)^{\alpha}\right)}\right. \\
& \left.\left.+\frac{21 \Gamma(1+2 \alpha)}{250-5^{3-2 \alpha} 16^{\alpha}}\right)\right) \\
& +\frac{t^{5 \alpha}}{\Gamma(5 \alpha+1)}\left(\frac{-437 \Gamma(1+4 \alpha)}{37,500(256)^{\alpha}(625)^{-\alpha}-75,000}\right. \\
& -\frac{(256)^{\alpha}-10(625)^{\alpha}}{5(256)^{\alpha}-10(625)^{\alpha}}\left(\frac{27 \Gamma(1+3 \alpha)}{625(64)^{\alpha}(125)^{-\alpha}-1250}\right. \\
& -\frac{\left(64^{\alpha}-10(125)^{\alpha}\right)}{5(64)^{\alpha}-10(125)^{\alpha}}\left(\frac{\left(64^{\alpha}-10(100)^{\alpha}\right)\left(-5(4)^{\alpha}(5)^{-\alpha}+50+14 \Gamma(1+\alpha)\right)}{125\left(2-4^{\alpha}(5)^{-\alpha}\right)\left(64^{\alpha}-2(100)^{\alpha}\right)}\right. \\
& \left.\left.\left.+\frac{21 \Gamma(1+2 \alpha)}{250-5^{3-2 \alpha} 16^{\alpha}}\right)\right)\right) \\
& +\frac{t^{6 \alpha}}{\Gamma(6 \alpha+1)}\left(\frac{-113 \Gamma(1+5 \alpha)}{37,500(1024)^{\alpha}(3125)^{-\alpha}-75,000}\right. \\
& -\frac{(1024)^{\alpha}-10(3125)^{\alpha}}{2(1024)^{\alpha}-5(3125)^{\alpha}}\left(\frac{-437 \Gamma(1+4 \alpha)}{37,500(256)^{\alpha}(625)^{-\alpha}-75,000}\right. \\
& -\frac{(256)^{\alpha}-10(625)^{\alpha}}{5(256)^{\alpha}-10(625)^{\alpha}}\left(\frac{27 \Gamma(1+3 \alpha)}{625(64)^{\alpha}(125)^{-\alpha}-1250}\right. \\
& -\frac{\left(64^{\alpha}-10(125)^{\alpha}\right)}{5(64)^{\alpha}-10(125)^{\alpha}}\left(\frac{\left(64^{\alpha}-10(100)^{\alpha}\right)\left(-5(4)^{\alpha}(5)^{-\alpha}+50+14 \Gamma(1+\alpha)\right)}{125\left(2-4^{\alpha}(5)^{-\alpha}\right)\left(64^{\alpha}-2(100)^{\alpha}\right)}\right. \\
& \left.\left.\left.\left.+\frac{21 \Gamma(1+2 \alpha)}{250-5^{3-2 \alpha} 16^{\alpha}}\right)\right)\right)\right) \text {. }
\end{aligned}
$$

The 6th approximate solution of Equation (34) when $\alpha=1$ will be

$$
g_{6}(t)=t-t^{2}+\frac{t^{3}}{2}-\frac{t^{4}}{6}+\frac{t^{5}}{24}+\frac{t^{6}}{120} .
$$

It is good to mention that the first sixth terms of the solution are the same in comparison of the first six terms in the series representation of the accurate solution $g(t)=t e^{-t}$ when $\alpha=1$.

Table 1 shows the absolute errors with respect to $\alpha=1$ with the 6 th and 16 th approximate ARA-RPS solution of the FNPEs in Equations (24) and (35).

We compare the obtained results by some methods, such as the two-stage orderone Runge-Kutta method and the variational iterative and the Chebyshev polynomial methods [33,38,39].

It can be concluded, according to Table 2, that the proposed method has less computational errors than the other mentioned methods, and the convergent of the ARA-RPS solution is a faster approach to the exact solution. 
Table 1. The absolute errors at $\alpha=1$ in different methods for Example 2.

\begin{tabular}{|c|c|c|c|c|c|}
\hline \multirow{2}{*}{$t$} & \multirow{2}{*}{$\begin{array}{c}\text { Two-Stage } \\
\text { Order-One } \\
\text { Runge-Kutta }\end{array}$} & \multirow{2}{*}{$\begin{array}{c}\text { Variational } \\
\text { Iterative }(k=6)\end{array}$} & \multirow{2}{*}{$\begin{array}{l}\text { Chebyshev } \\
\text { Polynomials } \\
\quad(k=16)\end{array}$} & \multicolumn{2}{|c|}{ ARA-RPS } \\
\hline & & & & $(k=6)$ & $(k=16)$ \\
\hline 0.2 & $1.49 \times 10^{-3}$ & $2.14 \times 10^{-3}$ & $5.24 \times 10^{-9}$ & $1.73 \times 10^{-8}$ & $1.05 \times 10^{-17}$ \\
\hline 0.4 & $2.16 \times 10^{-3}$ & $2.84 \times 10^{-3}$ & $3.38 \times 10^{-9}$ & $2.15 \times 10^{-6}$ & $4.71 \times 10^{-17}$ \\
\hline 0.6 & $2.31 \times 10^{-3}$ & $2.67 \times 10^{-3}$ & $3.12 \times 10^{-9}$ & $3.58 \times 10^{-5}$ & $5.27 \times 10^{-17}$ \\
\hline 0.8 & $2.17 \times 10^{-3}$ & $2.04 \times 10^{-3}$ & $8.41 \times 10^{-9}$ & $2.61 \times 10^{-4}$ & $1.05 \times 10^{-15}$ \\
\hline 1.0 & $1.68 \times 10^{-3}$ & $1.22 \times 10^{-3}$ & $5.43 \times 10^{-9}$ & $1.21 \times 10^{-3}$ & $4.51 \times 10^{-14}$ \\
\hline
\end{tabular}

Table 2. The residual errors at different $\alpha$ of the 6th approximate of ARA-RPS solution for Example 2.

\begin{tabular}{ccccc}
\hline $\boldsymbol{t}$ & $\boldsymbol{\alpha}=\mathbf{0 . 7 0}$ & $\boldsymbol{\alpha}=\mathbf{0 . 8}$ & $\boldsymbol{\alpha}=\mathbf{0 . 9}$ & $\boldsymbol{\alpha}=\mathbf{1 . 0}$ \\
\hline 0.2 & $2.42 \times 10^{-4}$ & $3.24 \times 10^{-5}$ & $4.2 \times 10^{-6}$ & $5.4 \times 10^{-7}$ \\
0.4 & $4.44 \times 10^{-4}$ & $9.03 \times 10^{-4}$ & $1.78 \times 10^{-4}$ & $3.45 \times 10^{-5}$ \\
0.6 & $2.44 \times 10^{-2}$ & $6.32 \times 10^{-3}$ & $1.58 \times 10^{-3}$ & $3.93 \times 10^{-4}$ \\
0.8 & $8.16 \times 10^{-2}$ & $2.51 \times 10^{-2}$ & $7.47 \times 10^{-3}$ & $2.20 \times 10^{-3}$ \\
1.0 & $2.08 \times 10^{-1}$ & $7.34 \times 10^{-2}$ & $2.49 \times 10^{-2}$ & $8.4 \times 10^{-3}$ \\
\hline
\end{tabular}

To introduce more comparisons according to the approximate ARA-RPS solution obtained in (38), we obtain the residual error of the 6th approximate ARA-RPS solution at diverse values of $t$ to show the exactness and effectiveness of the proposed method.

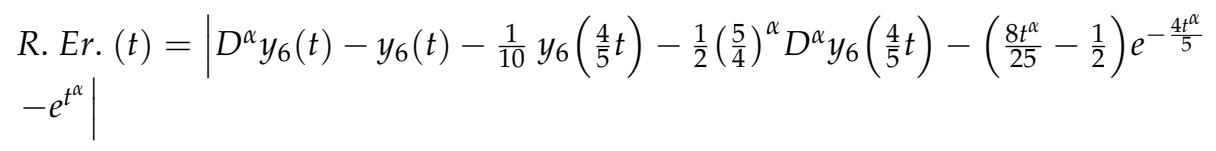

Figure 1 represents the graph of the first sixth terms of the approximate ARA-RPS solution of Example 2 for diverse values of $\alpha$. The effectiveness of the proposed method is obvious in the following figure, that is, with different values of $\alpha$, we have the graph of the ARA-RPS solution coincide with the exact solution when $\alpha=1$.

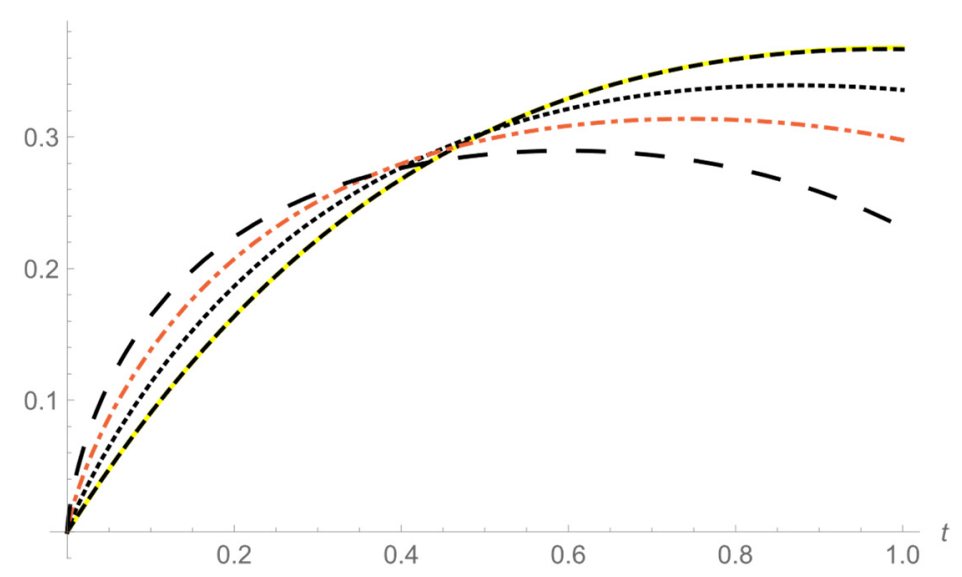

Figure 1. The behavior of the sixth approximate solution of Equations (34) and (35) for different values of $\alpha$. Solid line: exact solution at $\alpha=1$, dashed line: $g_{6}(t)$ at $\alpha=1$, dotted line: $g_{6}(t)$ at $\alpha=0.9$, dash. dotted line: $g_{6}(t)$ at $\alpha=0.8$, large dash. line: $g_{6}(t)$ at $\alpha=0.7$.

\section{Conclusions}

In the history of mathematics, there are several methods for solving differential equations, that have many applications in different fields of sciences and engineering. Recently, fractional differential equations have appeared, and many techniques have been developed to solve them. In this study, a combination of the ARA transform and the residual power series is implemented to solve fractional equations and other symmetric equations. 
The efficiency of the method was shown by presenting two numerical examples and comparing the obtained results with other methods.

As a future work, we attend to extend the use of the ARA-RPSM to solve autonomous n-dimensional fractional nonlinear systems and to construct an equivalent method to solve partial fractional differential equations.

Author Contributions: Data curation, R.S., A.B. and A.Q.; Formal analysis, A.B., R.S. and A.Q.; Investigation, A.B., R.S. and A.Q.; Methodology, A.B., R.S. and A.Q.; Project administration, A.Q., R.S. and A.B.; Resources, A.B., R.S. and A.Q.; Writing-Original draft, A.Q., R.S. and A.B.; WritingReview and editing, A.B., R.S. and A.Q. All authors have read and agreed to the published version of the manuscript.

Funding: This research is funded by the Deanship of Research at Zarqa University, Jordan.

Institutional Review Board Statement: Not applicable.

Informed Consent Statement: Not applicable.

Data Availability Statement: Not applicable.

Acknowledgments: The authors express their gratitude to the dear referees, who wish to remain anonymous, and the editor for their helpful suggestions, which improved the final version of this paper.

Conflicts of Interest: The authors declare no conflict of interest.

\section{References}

1. Qazza, A.; Hatamleh, R.; Alodat, N. About the Solution Stability of Volterra Integral Equation with Random Kernel. Far East J. Math. Sci. 2016, 100, 671-680. [CrossRef]

2. Pedrayes, J.F.; Melero, M.G.; Norniella, J.G.; Cabanas, M.F.; Orcajo, G.A.; González, A.S. Supercapacitors in Constant-Power Applications: Mathematical Analysis for the Calculation of Temperature. Appl. Sci. 2021, 11, 10153. [CrossRef]

3. Qazza, A.; Hatamleh, R. The existence of a solution for semi-linear abstract differential equations with infinite B chains of the characteristic sheaf. Int. J. Appl. Math. 2018, 31, 611-620. [CrossRef]

4. Arqub, O.A.; Al-Smadi, M.; Gdairi, R.A.; Alhodaly, M.; Hayat, T. Implementation of reproducing kernel Hilbert algorithm for pointwise numerical solvability of fractional Burgers' model in time-dependent variable domain regarding constraint boundary condition of Robin. Results Phys. 2021, 24, 104210. [CrossRef]

5. Ahmad, I.; Shah, K.; Rahman, G.U.; Baleanu, D. Stability analysis for a nonlinear coupled system of fractional hybrid delay differential equations. Math. Methods Appl. Sci. 2020, 43, 8669-8682. [CrossRef]

6. Yang, X.; Qiu, Y.; Zhang, M.; Zhang, L.; Li, H. Facile Fabrication of Polyaniline/Graphene Composite Fibers as Electrodes for Fiber-Shaped Supercapacitors. Appl. Sci. 2021, 11, 8690. [CrossRef]

7. Abu-Gdairi, R.; Hasan, S.; Al-Omari, S.; Al-Smadi, M.; Momani, S. Attractive Multistep Reproducing Kernel Approach for Solving Stiffness Differential Systems of Ordinary Differential Equations and Some Error Analysis. CMES 2021. in editing.

8. Oldham, K.; Spanier, J. The Fractional Calculus: Theory and Applications of Differentiation and Integration to Arbitrary Order; Academic Press: New York, NY, USA, 1974.

9. Podlubny, I. Fractional Differential Equations; Academic Press: San Diego, CA, USA, 1999.

10. Mainardi, F. Fractional Calculus and Waves in Linear Viscoelasticity; Imperial College Press: London, UK, 2010.

11. Almeida, R.; Tavares, D.; Torres, D. The Variable-Order Fractional Calculus of Variations; Springer: Cham, Switzerland, 2019.

12. Miller, K.; Ross, B. An Introduction to the Fractional Calculus and Fractional Differential Equations; Wiley: New York, NY, USA, 1993.

13. Adomian, G. Solving Frontier Problems of Physics: The Decomposition Method; Kluwer Academic Publisher: Boston, MA, USA, 1994.

14. Jafari, H.; Daftardar-Gejii, V. Solving linear and nonlinear fractional diffusion and wave equations by Adomian decomposition. Appl. Math. Comput. 2006, 180, 488-497. [CrossRef]

15. Saadeh, R. Numerical solutions of fractional convection-diffusion equation using finite-difference and finite-volume schemes. J. Math. Comput. Sci. 2021, 11, 7872-7891.

16. He, J.H. Homotopy perturbation technique. Comput. Methods Appl. Mech. Eng. 1999, 178, 257-262. [CrossRef]

17. Kashkari, B.S.; El-Tantawy, S.A.; Salas, A.H.; El-Sherif, L.S. Homotopy perturbation method for studying dissipative nonplanar solitons in an electronegative complex plasma. Chaos Solitons Fractals 2020, 130, 109457. [CrossRef]

18. Saadeh, R. Numerical algorithm to solve a coupled system of fractional order using a novel reproducing kernel method. Alex. Eng. J. 2021, 60, 4583-4591. [CrossRef]

19. Khandaqji, M.; Burqan, A. Results on sequential conformable fractional derivatives with applications. J. Comput. Anal. Appl. 2021, $29,1115-1125$. 
20. Abu-Gdairi, R.; Al-Smadi, M.; Gumah, G. An Expansion Iterative Technique for Handling Fractional Differential Equations Using Fractional Power Series Scheme. J. Math. Stat. 2015, 11, 29-38. [CrossRef]

21. Khaminsou, B.; Sudsutad, W.; Thaiprayoon, C.; Alzabut, J.; Pleumpreedaporn, S. Analysis of Impulsive Boundary Value Pantograph Problems via Caputo Proportional Fractional Derivative under Mittag-Leffler Functions. Fractal Fract. $2021,5,251$. [CrossRef]

22. Burqan, A.; El-Ajou, A.; Saadeh, R.; Al-Smadi, M. A new efficient technique using Laplace transforms and smooth expansions to construct a series solutionsto the time-fractional Navier-Stokes equations. Alex. Eng. J. 2021, in press.

23. Abbas, M.I.; Ragusa, M.A. On the Hybrid Fractional Differential Equations with Fractional Proportional Derivatives of a Function with Respect to a Certain Function. Symmetry 2021, 13, 264. [CrossRef]

24. El-Ajou, A.; Oqielat, M.; Al-Zhour, Z.; Momani, S. A class of linear non-homogenous higher order matrix fractional differential equations: Analytical solutions and new technique. Fract. Calc. Appl. Anal. 2020, 23, 356-377. [CrossRef]

25. Shqair, M.; El-Ajou, A.; Nairat, M. Analytical solution for multi-energy groups of neutron diffusion equations by a residual power series method. Mathematics 2019, 7, 633. [CrossRef]

26. Ahmad, M.; Asjad, M.; Singh, J. Application of novel fractional derivative to heat and mass transfer analysis for the slippage flow of viscous fluid with single-wall carbon nanotube subject to Newtonian heating. Math. Meth. Appl Sci. 2021, 1-16. [CrossRef]

27. Saadeh, R.; Qazza, A.; Burqan, A. A New Integral Transform: ARA Transform and Its Properties and Applications. Symmetry 2020, 12, 925. [CrossRef]

28. Qazza, A.; Burqan, A.; Saadeh, R. A New Attractive Method in Solving Families of Fractional Differential Equations by a New Transform. Mathematics 2021, 9, 3039. [CrossRef]

29. Mahmood, S.; Shah, R.; Khan, H.; Arif, M. Laplace Adomian decomposition method for multi-dimensional time fractional model of Navier-Stokes equation. Symmetry 2019, 11, 149. [CrossRef]

30. Kumar, D.; Singh, J.; Kumar, S. A fractional model of Navier-Stokes equation arising in unsteady flow of a viscous fluid. J. Assoc. Arab Univ. Basic Appl. Sci. 2015, 17, 14-19. [CrossRef]

31. Eriqat, T.; El-Ajou, A.; Moa'ath, N.O.; Al-Zhour, Z.; Momani, S. A new attractive analytic approach for solutions of linear and nonlinear neutral fractional pantograph equations. Chaos Solitons Fractals 2020, 138, 109957. [CrossRef]

32. Magin, R.L. Fractional calculus models of complex dynamics in biological tissues. Comput. Math. Appl. 2010, 59, 1586-1593. [CrossRef]

33. Sedaghat, S.; Ordokhani, Y.; Dehghan, M. Numerical solution of the delay differential equations of pantograph type via Chebyshev polynomials. Commun. Nonlinear Sci. Numer. Simul. 2012, 17, 4815-4830. [CrossRef]

34. Balachandran, K.; Kiruthika, S. Existence of solutions of nonlinear fractional pantograph equations. Acta Math. Sci. 2013, 33, 712-720. [CrossRef]

35. Bellen, A.; Zennaro, M. Numerical Methods for Delay Differential Equations. In Numerical Mathematics and Scientific Computation; The Clarendon Press: Oxford, UK; University Press: New York, NY, USA, 2003.

36. Chen, X.; Wang, L. The variational iteration method for solving a neutral functional-differential equation with proportional delays. Comput. Math. Appl. 2010, 59, 2696-2702. [CrossRef]

37. Rahimkhani, P.; Ordokhani, Y.; Babolian, E. Numerical solution of fractional pantograph differential equations by using generalized fractional-order Bernoulli wavelet. J. Comput. Appl. Math. 2017, 309, 493-510. [CrossRef]

38. Hashemi, M.S.; Atangana, A.; Hajikhah, S. Solving fractional pantograph delay equations by an effective computational method. Math. Comput. Simul. 2020, 177, 295-305. [CrossRef]

39. Ghasemi, M.; Fardi, M.; Ghaziani, R.K. Numerical solution of nonlinear delay differential equations of fractional order in reproducing kernel Hilbert space. Appl. Math. Comput. 2015, 268, 815-831. [CrossRef] 